\title{
Springer
}

Draft Manuscript for Review

\section{First evidence of the interaction between deleted in malignant brain tumor 1 and galectin-3 in the mammalian oviduct}

\begin{tabular}{|r|l|}
\hline Journal: & Histochemistry and Cell Biology \\
\hline Manuscript ID: & HCB-2707-13-Drenckhahn.R1 \\
\hline Manuscript Type: & Original manuscripts \\
\hline Date Submitted by the Author: & 20 -Aug-2013 \\
\hline Complete List of Authors: & $\begin{array}{l}\text { Roldán, María; Instituto de Biología Molecular y Celular de Rosario- } \\
\text { CONICET, } \\
\text { Marini, Patricia; Universidad Nacional de Rosario, Facultad de Ciencias } \\
\text { Bioquímicas y Farmacéuticas; Instituto de Biologia Molecular y Celular de } \\
\text { Rosario-CONICET, }\end{array}$ \\
\hline Keywords: & oviduct, Fallopian tube, DMBT1, galectin-3, cell polarization \\
\hline & \\
\hline
\end{tabular}


First evidence of the interaction between deleted in malignant brain tumor 1 and galectin-3 in the mammalian oviduct

\author{
M. L. Roldán ${ }^{1}$ and P. E. Marini ${ }^{1,2 *}$ \\ 1 Instituto de Biología Molecular y Celular de Rosario (IBR-CONICET) \\ 2 Área Biología, Facultad de Ciencias Bioquímicas y Farmacéuticas, UNR, Rosario, Argentina - Member of \\ the Research Career CIC-UNR \\ * Correspondence address: pmarini@ffbioyf.unr.edu.ar \\ Telephone: 54-341-4350661 ext122 \\ FAX: 54-341-4390465
}

\begin{abstract}
The oviduct supports the transport and final maturation of gametes, and harbors fertilization and early embryo development. The oviductal epithelium is responsible of providing the correct environment for these processes. Deleted in malignant brain tumor 1 (DMBT1) is expressed by multiple organisms and several cell types, and the interaction of the rabbit ortholog of DMBT1 with galectin-3 (gal-3) modulates the polarity of epithelial cells. DMBT1 promoted terminal differentiation has been proposed to occur in many epithelia, however This interaction between the components of this mechanism has not yet been shown in locations other than rabbit kidney and human cultured endothelial cells. DMBT1 and gal-3 also protect epithelial layers from pathogens and trauma, and are innate immunity components. DMBT1 has been detected in the porcine oviduct, and gal-3 has been reported in the Fallopian tube and in the cow oviduct. Interaction between both proteins would show a probable physiological function in the female reproductive tract be essential for cell terminal differentiation through the DMBT1 promoted mechanism. This work describes the presence and colocalization of DMBT1 and gal-3 mainly in the apical region of the epithelial cells of the Fallopian tube and the porcine oviduct, and co-immunoprecipitation in membrane enriched epithelial cell extracts from the porcine oviduct. The findings strongly support a functional interaction eompatible with cell differentiation in
\end{abstract}


the mammalian oviduct, suggestive of a role on epithelial protection and homeostasis, which might be related to epithelium-gamete interaction.

Keywords: oviduct - Fallopian tube - DMBT1 - galectin-3

\section{Introduction}

The mammalian oviduct is the site where the final steps of gamete maturation and fertilization take place, and where the initial stages of embryo development occur (Harper 1994). The characteristics of the cells that compose the oviductal epithelium are important for their direct interaction with the gametes and embryo, and determine the composition of the milieu that surrounds them. The microenvironment of the Fallopian tube influences gamete functions and interaction (Zumoffen et al. 2013), and affects the rate and outcome of fertilization (Schwarzer et al. 2012). Also, alterations in the Fallopian tube epithelial cells have been related to abnormal consequences, as tubal ectopic pregnancy (Gebeh et al. 2012, Ji et al. 2013).

The epithelium is the most abundant tissue of multicellular organisms and its cells come from stem cells that have a two steps differentiation. The first step consists on the development of apical and basolateral membrane domains and the formation of sheets of cells connected by junctions, forming the protoepithelia. The second step, called terminal differentiation, is tissue and organ specific and renders the mature phenotype. This process continues to occur in adult animals in the intestine, skin, prostate, and other organs. The mechanism underlying the second step of epithelial cells differentiation has been analyzed mainly in rabbit kidney intercalated cells, showing both in vivo and in vitro a central role for hensin, the cunic ortholog of deleted in malignant brain tumor 1 (DMBT1) (for review see Al-Awqati and Gao 2011). In this differentiation mechanism DMBT1 suffers undergoes galectin-3 (gal-3) mediated polymerization. DMBT1 deposits bind to integrins and produce the conversion of the epithelial cells to a cuboidal phenotype (AlAwqati 2011). This mechanism has been considered an example of terminal differentiation and, as DMBT1 is expressed in most epithelia, often in alternately spliced forms, it has been proposed to be a ubiquitous pathway. This proposal is supported by early embryonic lethality at the time of appearance of the first columnar epithelium, visceral-endoderm, in mouse with global DMBT1 deletion (Takito and Al-Awqati 2004), and also by the relationship between the relationship between DMBT1's deletion or down-regulation 
is related to malignancy in a large number of epithelia (see Dodurga et al. 2011 for review), and as malignancy is often associated to lack of differentiation, this supports the proposal for ubiquity of DMBT1 dependent differentiation among epithelial tissues. Recently, we have identified DMBT1 in the porcine oviduct in the context of a process of negative selection of sperm, which may be related to the control of polyspermia (Teijeiro et al. 2008, 2011, 2012, Teijeiro and Marini, 2012). DMBT1 has also been detected in porcine oocytes (Ambrousi et al. 2013), opening the possibility of other functions for the glycoprotein in the complex reproductive process.

DMBT1 has been assigned dual functions in innate immunity and epithelial differentiation/cancer (Madsen et al. 2010). It is considered to protect epithelial layers from potential pathogens and trauma, coordinating the cell functions related to differentiation/cancer with those of innate immunity (Madsen et al. 2010). It is also considered a pH dependent Golgi-sorting receptor in the exocrine pancreas (Boulatnikov and De Lisle 2004). Recently, we have identified DMBT1 in the porcine oviduct in the context of a process of negative selection of sperm, which may be related to the control of polyspermia (Teijeiro et al. 2008, 2011, 2012, Teijeiro and Marini, 2012). DMBT1 has also been detected in porcine oocytes (Ambrousi et al. 2013), opening the possibility of other functions for the glycoprotein in the complex reproductive process.

Gal-3 is another protein related to innate immunity, development and tumour progression. Gal-3 is the most widely studied member of the galectin family and is found in various cell types including monocytes, macrophages and epithelial cells. It may localize to cell surfaces, the extracellular matrix, the cytoplasm and the nucleus (Dumic et al. 2006), and its functions are classified by location. Specific interactions of gal-3 with a variety of intra- and extracellular proteins affect numerous biological processes related to physiological and pathophysiological conditions such as development, immune reactions, neoplastic transformation and metastasis. Gal-3 is also involved in the $\mathrm{pH}$ dependent subcellular targeting of apical glycoproteins by membrane recycling (Straube et al. 2013). In the female reproductive tract it has been reported in cow (Kim et al. 2008) and in human endometrial tissues, where it has been related to hyper and neoplasia (Brustmann et al 2003).

Being The correct composition of the Fallopian tube epithelium is critical for its role in fertilization and its effects over gametes and early embryo development. Thus, it is tempting to investigate the possible involvement of DMBT1 and gal-3 in oviductal cells polarization/differentiation or in protective functions. 
The terminal differentiation mechanism-sustained for rabbit kidney (A1-Awqati and Gro 2011), endothelial eells (Müller et al. 2012) and intestine (Hikita et al. 2000) involves interaction with gal-3-Gal-3 is the most widely studied member of the galectin family and is found in various cell types including monocytes, macrophages and epithelial cells. In the female reproductive tract it has been reported in cow (Kim et al. 2008) and in human endometrial tissues, where it has been related to hyper and neoplasia (Brustmann et al 2003).

In order to analyze the hypothesis that DMBT1 and gal-3 determine coordinated biological functions dependent cell differentiation might occur in the mammalian oviduct, we begin by searching for their possible analyzing DMBT1 and gal-3 interaction. DMBT1 is reported for the first time in the Fallopian tube, and gal-3 in the porcine oviduct. Co-localization of both proteins is assayed in both species, and coimmunoprecipitation is obtained for the porcine oviduct. Our results are a first evidence of the DMBT1 and gal-3 functional interaction dependent differentiation mechanism-in the mammalian oviduct.

\section{Materials and Methods}

\section{Chemicals and antibodies}

Unless otherwise stated, chemicals were obtained from Sigma- Aldrich, Argentina.

Anti-gal-3 serum developed in goat was gently provided by Dr. Fu-Tong Liu (University of California, USA). For DMBT1 detection anti-CUB antibodies developed in this work were used. Epithelial cells from the isthmic region of the porcine oviduct were obtained by scrapping with a scalpel blade in TRIzol-reagent (Invitrogen, Argentina). Total RNA was isolated and $5 \mu \mathrm{g}$ aliquots reverse transcribed by oligodT using SuperScript-II reverse transcriptase (Invitrogen, Argentina). The cDNA $(1 \mu l)$ was used as template in PCR in a Mastercycler thermocycler (Eppendorf, Germany). The DMBT1 primers sequences were: 5'-ATCGGATCCCGGTTTGGTCAGGGCTCA-3' and 5'-TTGAATTCCATCGGCCACCTTGGTA-3'. The oligonucleotides were designed with BamHI (sense) and EcoRI (antisense) restriction sites (in italics) to facilitate posterior cloning in pGEX-2T, which encodes GST-tag at the $\mathrm{NH}_{2}$ terminus. The amplified product was cloned, and sequenced at Maine University. Expression of the cloned GST-tagged CUB domain from porcine DMBT1 in Escherichia coli (strain DH5 $\alpha$ ) was induced by $1 \mathrm{mM}$ IPTG at $37{ }^{\circ} \mathrm{C}$ for $4 \mathrm{~h}$. Recombinant protein was used to generate polyclonal anti-CUB antibodies in rabbits as previously reported 
(Pérez et al. 2006) and according to the protocol approved by Facultad de Ciencias Bioquímicas y Farmacéuticas, UNR. Control experiments using preimmunization serum, anti-GST or secondary antibodies alone were done (data not shown). On immunoblots, the developed antibodies show specificity comparable to the described for other anti-porcine DMBT1 antibodies (Pérez et al. 2006, Teijeiro et al. 2012) and antiDMBT1p84 (polyclonal rabbit antibody against human DMBT1 anti-human DMBT1 antibodies (antihDMBT1 - gently provided by Dr. Jan Mollenhauer, University of Southern Denmark, Denmark) (Müller et al., 2007 Renner et al. 2007), upon examination of porcine tissues.

For western blot and immunohistochemistry, anti-rabbit IgG-HRP from GE Healthcare and anti-goat IgGHRP from Santa Cruz Biotechnology, Argentina, were used. For immunofluorescence, cy2-conjugated donkey anti-rabbit IgG (Jackson Immuno Research Laboratories, Inc, USA) and cy3-conjugated rabbit antigoat IgG from Chemicon, Millipore, Argentina, were also used.

\section{Samples}

For human samples, study protocols were approved by the Institutional Bioethical Board of the Facultad de Ciencias Bioquímicas y Farmacéuticas, Universidad Nacional de Rosario, and a written consent was obtained from all donors. Human oviductal tissue was obtained from premenopausal women with no clinical history of infection or neoplasic diseases, scheduled for hysterectomies as a result of uterine fibromyomas or hypermenorrea (Hospital Provincial del Centenario, Rosario, Argentina). No patient received any hormonal treatment prior to hysterectomy.

Porcine oviducts were obtained at a local abattoir and transported to the laboratory in ice-cold PBS.

\section{Western blot}

Fragments of Fallopian tubes and porcine oviducts were open longitudinally, and the epithelial layer of cells was carefully scraped out with a scalpel and homogenized in homogenization buffer [50 $\mathrm{mM}$ Tris- $\mathrm{HCl}$ (pH 7.4), 0.1 mM EDTA, 0.1 mM EGTA, 0.1 M Phenylmethanesulfonyl fluoride (PMSF), $2 \mu \mathrm{g} / \mathrm{ml}$ Aprotinin and $0.1 \% \mathrm{v} / \mathrm{v} 2$-mercaptoethanol] in an ice bath. The oviduct cell homogenates were then centrifuged at $15,000 \mathrm{~g}$ for $30 \mathrm{~min}$ at $4^{\circ} \mathrm{C}$, and the supernatant was stored at $-20^{\circ} \mathrm{C}$ until further use (Zumoffen et al. 2013). 
Total protein concentration in oviductal cell homogenates was assessed with the Bradford Protein assay (Bradford 1976).

Extracts (50 $\mu \mathrm{g}$ of proteins) were subjected to SDS-PAGE and transferred to nitrocellulose membranes (GE Healthcare, Rosario, Argentina). Membranes were blocked with 5\% dry milk in TBS-T (TBS plus $0.05 \%$ Tween-20) for $2 \mathrm{~h}$ and incubated with rabbit anti-CUB (1:1000), anti-hDMBT1p84 (1:1000) or goat anti-gal-3 (1:1000) serum during $2 \mathrm{~h}$ at room temperature. After washing with TBS, they were incubated with anti-rabbit IgG-HRP or anti-goat IgG-HRP respectively, 1:10,000 (v/v) in TBS, during $1 \mathrm{~h}$ at room temperature, and washed again with TBS. Peroxidase activity was revealed using ECL kit (GE Healthcare, Argentina) according to the manufacturer's instructions.

\section{Immunohistochemistry and co-immunolocalization of DMBT1 and galectin-3 in oviductal tissues}

The human and gilt's oviducts were separated by dissection into $1-\mathrm{cm}$ segments and fixed in $4 \%$ formaldehyde. Tissue was dehydrated, embedded in paraffin, cut into $5-\mu \mathrm{m}$ sections, and mounted on slides optimized for immunohistochemistry (Frosted HiFixNH, TNT, Argentina). Slides were dewaxed twice in xylene $100 \%$ for 10 minutes and rehydrated in decreasing concentrations of ethanol $(100 \%, 96 \%, 70 \%$ and $35 \%$ ) during 5 minutes, each step. For immunohistochemistry endogenous peroxidases were inactivated by incubating slides for $30 \mathrm{~min}$ in $3 \% \mathrm{~V} / \mathrm{V} \mathrm{H}_{2} \mathrm{O}_{2}$ in methanol. Antigen retrieval was done with pre-warmed $10 \mathrm{mM}$ sodium citrate $\mathrm{pH}$ 6.0, followed by three 5 min washes in TBS. Sections were blocked with $0.05 \%$ Tween-20, 5\% dry non-fat milk in TBS for $60 \mathrm{~min}$ at room temperature, and then treated with anti-CUB or anti-hDMBT1p84 (1:25) or anti-gal-3 (1:50) antibodies overnight at $4^{\circ} \mathrm{C}$ followed by anti-rabbit or anti-goat IgG-HRP (diluted 1:100). Bound antibody was visualized by development with 3,3'-diaminobenzidine tetrahydrochloride (DAB), stopping the reaction by washing in water. The sections were counterstained with haematoxylin. In negative controls, the primary antibody was omitted or replaced by pre-immune serum.

For co-localization experiments, after antigen retrieval and rinsing for 5 minutes in running water, the slides were treated with $5 \%$ BSA in PBS to minimize nonspecific binding before incubating overnight at $4{ }^{\circ} \mathrm{C}$ with rabbit anti-CUB (1:25) and goat anti-gal-3 (1:50) antibodies. Slides were rinsed with PBS and incubated $2 \mathrm{~h}$ with cy2- conjugated donkey anti-rabbit IgG at a dilution of 1:100 in 3\% BSA, PBS. After rinsing with PBS, 
the slides were incubated with cy3 conjugated rabbit anti-goat IgG for $2 \mathrm{~h}$ (1:100 dilution in 1\% BSA, PBS), rinsed with PBS and then incubated with Hoescht 33258 for 3 minutes. Finally, the slides were washed with PBS three times, covered with DABCO solution (1\% phenylenediamine in glycerol:PBS, 5:1), and coverslips were added. Preparations were examined with a confocal microscope (Nikon Model Eclipse TE-2000-E2, USA). Controls were run by omission of the primary antibody and by adding rabbit pre-immunization serum.

\section{Immunoprecipitation}

Porcine oviductal epithelial cell fractions enriched in plasmatic membranes were obtained as described in Marini and Cabada (2003). Briefly, oviducts of prepubertal gilts of approximately 120 days of age were used. The isthmic part was separated and opened longitudinally. Epithelial cells were obtained by scrapping with the blunt side of a scalpel blade, disaggregated by passing through a 21 gauge needle to separate the cells, which were disrupted using Potter homogenizer. Fractions enriched in plasma membrane were prepared by differential centrifugation. Extracts of these fractions were obtained by incubation in $0.5 \mathrm{M} \mathrm{NaCl}, 0.2 \%$ Triton $\mathrm{X} 100,10 \mathrm{mM}$ Tris- $\mathrm{HCl} \mathrm{pH} 7.5$ for $1 \mathrm{~h}$ at $4^{\circ} \mathrm{C}$.

Rabbit anti-CUB or goat anti-gal-3 serum was added to porcine oviduct extracts containing $1 \mathrm{mg}$ of protein, at a dilution of 1:10 and 1:25, respectively, and immunoprecipitation was performed at $4{ }^{\circ} \mathrm{C}$ overnight. Immunoprecipitates obtained using anti-CUB or anti-gal-3 antibodies were collected by mixing with protein A/G-Agarose (Thermo Scientific, Argentina), at $4{ }^{\circ} \mathrm{C}$ for $1 \mathrm{~h}$. Beads were washed three times following manufacturer's protocol. Immunoprecipitates were recovered from the beads by boiling in sample buffer for 5 min, and then they were subjected to SDS-PAGE (4-15\% gradient). Proteins were transferred to nitrocellulose membranes and submitted to western blot as describe above.

\section{RESULTS}

\section{Detection and localization of DMBT1 in the Fallopian tube}

The presence of DMBT1 has not been described in the Fallopian tube. To analyze if the glycoprotein is present in this organ, western blot experiments were conducted on extracts from human oviducts using antiCUB and anti-hDMBT1p84 antibodies. The results shown in Fig. 1 a, indicate that both antibodies recognize a protein of the expected apparent molecular mass in human oviductal extracts. 
The localization of the protein was analyzed by immunohistochemistry with anti-CUB and anti-hDMBT1p 84 antibodies and DAB staining. Again, both antibodies showed the same result, intense immunoreactivity at the apical region of the epithelial cells lining the tube (Fig. $1 \mathrm{~b}, \mathrm{c}$ ). Controls using pre-immunization serum gave no signal (Fig. 1 d).

\section{Expression of gal-3 in the porcine oviduct}

As the presence of gal-3 has been reported in cow and human oviducts but not in porcine oviducts, we assessed its expression by western blot of oviductal cell extracts. The expected $31 \mathrm{kDa}$ protein was detected (Fig. 2 a).

The localization of gal-3 in the porcine oviducts was analyzed by immunohistochemistry with anti-gal-3 antibodies, showing preferential DAB staining at the apical surface of the cells and occasional nuclear staining (Fig. 2 b). Controls without primary antibody showed no signal (Fig. 2 c)

\section{Co-localization experiments in the porcine oviduct and the Fallopian tube}

Interaction between DMBT1 and gal-3 is required for their engagement in a coordinated cellular function-cell differentiation and, for it to occur, both proteins should localize at the appropriated sites of the epithelial cells. The localization of DMBT1 in the Fallopian tube (Fig $1 \mathrm{~b}, \mathrm{c}$ ) is coincident with the one previously described for gal-3 (John et al. 2002). To analyze co-localization, fluorescent immunohistochemistry for both proteins was performed on the same sample, under the same conditions. Fluorescence staining was chosen for greater sensibility. The results for DMBT1 show again intense staining at the apical surface and also lighter, diffuse staining distributed over the entire cytoplasm, being more prominent in the luminal side of epithelial cells (Fig. 3 b). Cells present near the lumen and at the crypt's bases were stained (Fig. 3 b). The expression pattern of gal-3 (Fig. 3 c) showed substantial overlap with that found for DMBT1 (Fig. 3 d, e). For gal-3, but not for DMBT1, occasional nuclear staining of cells was observed (Fig. 3 c). Negative controls exhibited no signal (Fig. $3 \mathrm{~m}$ ).

Co-localization was also analyzed for DMBT1 and gal-3 in porcine oviducts by immunofluorescence with the corresponding antibodies. Again fluorescence was observed mainly at the apical region of the cells, with lighter staining of cytoplasms on all the lining cells for DMBT1 (Fig. $3 \mathrm{~h}$ ), in accordance with the previously 
described (Perez et al., 2006), except that staining of the crypt's bases (Fig. 3 h) had not been reported, probably due to method's sensibility. Overlapping staining for DMBT1 and gal-3 (Fig. 3 i) was detected in the oviducts of gilts (Fig. 3 j, k), as it was in Fallopian tubes (Fig. 3 d, e). Again, nuclear staining was detected for gal-3 (Fig. 3 i). Negative controls showed no signal (Fig. 3 n).

\section{Interaction between DMBT1 and Gal3 in the porcine oviduct}

To establish if there is actual interaction between DMBT1 and gal-3 in the oviductal epithelium, immunoprecipitation was performed using porcine oviductal cell extracts. Only the porcine model was used to perform these experiments due to the lack of sufficient amounts of human Fallopian tube extracts. Immunoprecipitation with anti-CUB antibodies, followed by western-blot detection of precipitated proteins with anti-gal-3 antibodies showed the presence of gal-3 (Fig. 4 a). Conversely, immunoprecipitation with antigal-3 antibodies followed by western-blot of the precipitated proteins with anti-CUB showed the presence of DMBT1 (Fig. 4 b). To exclude a putative galectin-3 binding to glycans from a-CUB antibodies, a control experiment was performed by using the cytoplasmic fraction obtained according to Marini et al., 2003, instead of membrane enriched fractions. No significant amount of gal-3 was found to precipitate (data not shown).Controls were also done with preimmune serum (data not shown).

\section{Discussion}

DMBT1 exists in different spliced forms that are either membrane-associated or secreted epithelial products. The functions proposed for DMBT1 include roles in innate immune defence and inflammation, epithelial differentiation and tumour suppression, and being a Golgi-sorting receptor in the exocrine pancreas (DeLisle et al. 2008). DMBT1 is the main component of the mechanism of terminal differentiation of epithelial cells, which has been shown for rabbit kidney intercalated cells, and has been proposed to be ubiquitous. DMBT1 has been reported as an estrogen and progesterone expression dependent protein in monkey's and rat's endometrium (Ace and Okulicz 2005; Tynan et al. 2005) and in the human genital tract, in relation to immunity and infection (Stoddard et al. 2007). Gal-3 has been described to mediate developmental processes, including cell differentiation, tissue organization, cell polarization and, more recently, regulation of the 
immune response (Vasta et al. 2012). The expression of this protein has been reported in the Fallopian tube (John et al. 2002) and in cow genital tract (Kim et al. 2008). Interaction between DMBT1 and gal-3 has been well established in rabbit kidney, as essential for terminal differentiation (Al-Awqati 2011), and in angiogenesis (Müller et al. 2012).

The objective of this study was to analyze the possibility that the characterized physiological functions described for DMBT1 and gal-3 in other tissues dependent epithelial cell differentiation mechanism-occurs in the Fallopian tube. In this organ, where epithelial cell's differentiation is related to the correct composition of the ergan's milieu, necessary for maternal-gamete and maternal-embryo productive interaction (Hunter 2012). These interactions also concern immunological aspects, related to tolerance to sperm and embryos, and sperm selection. The immunological roles assigned to DMBT1 (Madsen et al. 2010) and gal-3 in other localizations might as well be present in the Fallopian tube, which is a sterile organ.

The mentioned differentiation mechanism related to DMBT1 has been extensively studied in rabbit kidney, showing that the rabbit ortholog of DMBT1 (hensin) is secreted from the cells and deposited by polymerization in the extracellular matrix to become active (Hikita et al. 2000). The polymerization step is mediated mainly by gal-3 and is followed by inside-out integrin signaling, rendering a cuboidal phenotype of epithelial cells (Vijayakumar et al. 2008). The mechanism of DMBT1 and gal-3 excretion is still under study (Straube et al. 2013).

To begin analyzinge the hypothesis-In this work, we searched for DMBT1 in the Fallopian tube, with positive results (Fig. 1 a). The expression of DMBT1 in this organ had only been described in porcine (Marini and Cabada 2003, Teijeiro et al. 2012), where its localization is at the apical surface of epithelial cells (Pérez et al. 2006). Coincidently, in the Fallopian tube, immunostaining for DMBT1 was also found at the apical surface of the epithelial cells, but also in the cytoplasm, predominantly in the luminal side (Fig. 1 b and Fig. 3 b). The difference between both observations may be related to the greater sensibility of fluorescent staining. Recent results confirm the expression of DMBT1 in the cytoplasm as well as the apical region of porcine oviductal epithelial cells (Ambrousi et al. 2013). The described distribution is also coincident with that of hensin in rabbit kidney (Vijayakumar et al. 1999). No difference was noted on the distribution of DMBT1 between the crypt's bases and luminal regions of the tube, similar to the detected on mouse and rabbit's intestine (De Lisle et al. 1998, Takito et al. 1999). DMBT1 was also detected at the basal periphery of some porcine oviductal 
cells, in coincidence with the described by Mollenhauer et al. (2001) for monolayer and duct epithelia, pointing to secretion into the extracellular matrix. This localization would be compatible with a role in epithelial cell differentiation; however, basal location was not predominant.

Gal-3, another key component of the DMBT1 mediated differentiation mechanism, has been previously detected in human Fallopian tube, in relation to gonococcal invasion (John et al. 2002). In their study, the authors reported selective expression of gal-3 on the apical side of non-ciliated cells, detected by immunohistochemistry. Gal-3 has also been reported in the oviduct of cows (Kim et al. 2008) and, in this work, we show its presence and localization in pig's oviduct (Fig. 2). The localization at the surface of the epithelial cells is coincident in all the analyzed species.

The presence of the DMBT1 differentiation mechanism involved proteins, gal-3 and DMBT1, in the appropriated location (the apical surface of the cells) in oviductal epithelial cells has been described in this (Fig. 1, 2) and previous works (John et al. 2002, Pérez et al. 2006, Kim et al. 2008). However, co-localization needed to be challenged for each species, and under the same experimental conditions. Using immunofluorescence, both proteins were shown to localize coincidently in the surface of the epithelial cells in porcine and human Fallopian tube, being gal-3 also present in the nuclei (Fig. 3).

As a part of the DMBT1 differentiation mechanism gal-3 and DMBT1 are exported by a non-canonical mechanism, and DMBT1 polymerization is promoted by gal-3 in the extracellular matrix (Vijayakumar et al. 2008). In order for this to occur, both proteins should interact under physiological conditions. As gal-3 is a lectin with specificity for (Galß1-1/3GlcNAc)n (Bachhawat-skider et al. 2001, Rossez et al. 2011), DMBT1 exposes these saccharides (Marini and Cabada 2003, Rossez et al., 2011), and both proteins are found at the same localization, this interaction could be expected. Experiments to analyze if the interaction actually occurs in the oviduct were conducted in the porcine model, taking advantage of the facility of obtaining oviductal extracts in bigger amounts. Co-immunoprecipitation was obtained as a clear evidence of the interaction between DMBT1 and gal-3 (Fig. 4). It is important to notice that immunoprecipitation performed with antiCUB antibodies consistently rendered apparent greater amounts of gal-3 than immunoprecipitation with antigal-3 gave of DMBT1 (Fig. 4). This could be a reflection of the capacity of gal-3 to bind to multiple ligands in this location while DMBT1 probably binds predominantly to gal-3. The interaction found in the porcine 
model, together with co-localization of the proteins in the Fallopian tube, allows inferring a similar interplay in the human oviduct.

It is alse to note that co-immunoprecipitation of DMBT1 and gal-3 was obtained from plasma membrane enriched fractions (Fig. 4), and that co-localization was prevalent at the apical surface of the cells (Fig. 3). A role in cellular terminal differentiation similar to what occurs in rabbit kidney, would require showing that both proteins to interact only in the extracellular matrix, however the proteins were only occasionally detected at this location. The low prevalence of a basal targeting of DMBT1 and gal-3 indicates they are unlikely to participate in constitutive processes of cell differentiation, as stated by Mollenhauer and coworkers, 2001. Instead, localization prevalently at the apical surface together with pull-down from but also in membrane fractions, is in accordance with the apical protein sorting role proposed for gal-3 (Straube et al. 2013). MUC1, the dog ortholog of DMBT1, is considered an apical targeting signal in canine kidney cells, where gal-3 is thought to play the key role of cross-linking cargo in trans-Golgi network-derived vesicles (Kinlough et al. 2011). Hensin has been also described to switch the polarity of kidney epithelial cells (AlAwqati and Gao 2011). This possible role for DMBT1/gal-3 interaction in the oviduct is also supported by the observation of relative different amounts of co-immunoprecipitation using anti-CUB and anti-gal-3 antibodies (Fig. 4), which are in accordance with gal-3 binding to a larger amount of ligands. Once delivered to the apical surface DMBT1 may be engaged in epithelial protection and/or maintenance of epithelial integrity, as well as in epithelium-gamete interactions. Exosomes may derive from the oviductal epithelium containing DMBT1 and gal-3, which might interact with sperm, similarly to gal-3 containing prostasomes (Block et al. 2011).

The evidence presented here strongly supports the hypothesis that a biological function for DMBT1 and gal-3 interaction dependent differentiation might oecur in the epithelium of the Fallopian tube and the porcine oviduct. The detection and localization of gal-3 in the oviduct of cow (Kim et al. 2008) allows further hypothesizing that the mechanism may be extended among other mammals.

It is interesting to note that the expression of gal-3 and DMBT1 have been related to the menstrual cycle in the endometrial cells of human, rodents and primates (Ace and Okulicz 1998, von Wolff et al. 2005, Tynan et al. 2005, Yang et al. 2012), raising the possibility of a role related to a specific time in the hormonal cycle in the endometrium cyclic proliferation or differentiation and during pregnancy establishment, with putative 
implications for tumer suppressive and on mucosal protectionve functions (Tynan et al. 2005). The possible hormone dependent expression of DMBT1 in the oviduct, particularly in pigs, where polispermy is frequent, might be a link between sperm selection and specific moments of the estrus cycle.

The results presented here represent new evidence supporting DMBT1 and gal-3 possible involvement in the homeostasis differentiation of the epithelia covering the female tract, with possible implications in tumor progression. It also suggests DMBT1/gal-3 interaction related-oviductal-cell-differentiation may be a generalized mechanism extended among mammalian female reproductive tracts.

\section{Acknowledgements}

This study was supported by Agencia Nacional de Promoción de la Ciencia y Tecnología - BID program (grant number PICT 1284-2007) of Argentina, and grant 21010-098-12 from Secretaría de Estado de Ciencia, Tecnología e Innovación de la Provincia de Santa Fe, Argentina. We thank Dr. Fu-Tong Liu for anti-galectin3 antibodies, Dr. Jan Mollenhauer for anti-human-DMBT1p84 antibodies and Dr. Sergio Ghersevich for Fallopian tube samples. We also thank Frigorífico Paladini SA for providing gilt's oviducts.

\section{References}

Ace C, Okulicz W (1998) A progesterone-induced endometrial homolog of a new candidate tumor suppressor, DMBT1. J Clin Endocrinol Metab 83:3569-3573

Ace C, Okulicz W (2005) The 5' flanking region of the Rhesus monkey H3 (DMBT1) gene contains putative progesterone response elements. DNA Cell Biol 24:345-349

Al-Awqati Q (2011) Terminal differentiation in epithelia: the role of integrins in hensin polymerization. Annu Rev Phisiol 73:401-412

Al-Awqati Q, Gao X (2011) Differentiation of intercalated cells in the kidney. Physiology (Bethesda) 26:266272

Ambruosi B, Accogli G, Douet C, Canepa S, Pascal G, Monget P, Moros Nicolas C, Holmskov U, Mollenhauer J, Robbe-Masselot C, Vidal O, Desantis S, Goudet G (2013) Deleted in malignant brain tumour 
1 (DMBT1) is secreted in the oviduct and involved in the mechanism of fertilization in equine and porcine species. Reproduction 146:119-133

Bachhawat-Sikder K, Thomas CJ, Surolia A (2001) Thermodynamic analysis of the binding of galactose and poly-N-acetyllactosamine derivatives to human galectin-3. FEBS Lett 500:75-79

Block A, Saraswati S, Lichti C, Mahadevan M, Diekman A (2011) Co-Purification of Mac-2 Binding Protein with Galectin-3 and Association with Prostasomes in Human Semen. Prostate 71:711-721

Boulatnikov I, De Lisle R (2004) Binding of the Golgi sorting receptor Muclin to pancreatic zymogens through sulfated O-linked oligosaccharides. J Biol Chem 279:40918-40926

Bradford M (1976) Rapid and sensitive method for the quantitation of microgram quantities of protein utilizing the principle of protein-dye binding. Anal Biochem 72:248-254

Brustmann H, Riss D, Naudé S (2003) Galectin-3 expression in normal, hyperplastic, and neoplastic endometrial tissues. Pathol Res Pract 199:151-158

De Lisle RC, Petitt M, Isom KS, Ziemer D (1998) Developmental expression of a mucinlike glycoprotein (MUCLIN) in pancreas and small intestine of CF mice. Am J Physiol 275:G219-227

De Lisle R, Xu W, Roe B, Ziemer D (2008) Effects of Muclin (Dmbt1) deficiency on the gastrointestinal system. Am J Physiol Gastrointest Liver Physiol 294:G717-727

Dodurga Y, Avci C, Yilmaz S, Dogan Z, Kesen Z, Tataroglu C, Satiroglu-Tufan NL, Bushra T, Gunduz C (2011) Evaluation of deleted in malignant brain tumors 1 (DMBT1) gene expression in bladder carcinoma cases: preliminary study. Biomarkers 16:610-615

Dumic J, Dabelic S, Flogel M (2006) Galectin-3: an open-ended story. Biochem Biophys Acta 1760: 616-635

Gebeh A, Willets J, Marczylo E, Taylor A, K J (2012) Ectopic pregnancy is associated with high anandamide levels and aberrant expression of FAAH and CB1 in fallopian tubes. J Clin Endocrinol Metab 97:2827-2835 
Harper M (1994) Gamete and zygote transport. In: Knobil E, Neill J, (Eds). The physiology of reproduction. Raven Press, New York, USA, Pp123-187, 1994

Hikita C, Vijayakumar S, Takito J, Erdjument-Bromage H, Tempst P, Al-Awqati Q (2000) Induction of Terminal Differentiation in Epithelial Cells Requires Polymerization of Hensin by Galectin 3. J Cell Biol 151: $1235-1246$

Hunter R (2012) Components of oviduct physiology in eutherian mammals. Biol Rev Camb Philos Soc $87: 244-255$

Ji Y, Chen L, Xu K, Yao J, Shi Y, Shanguan X (2013) Reduced expression of aquaporin 9 in tubal ectopic pregnancy. J Mol Histol 44:167-173

John C, Jarvis G, Swanson K, Leffler H, Cooper M, Huflejt M, Griffiss J (2002) Galectin-3 binds lactosaminylated lipooligosaccharides from Neisseria gonorrhoeae and is selectively expressed by mucosal epithelial cells that are infected. Cell Microbiol 4:649-62

Kim M, Kim S, Kim H, Kim H, Joo HG, Shin T (2008) Immunohistochemical localization of galectin-3 in the reproductive organs of the cow. Acta Histochem 110:473-480

Kinlough C, Poland P, Gendler S, Mattila P, Mo D, Weisz O, Hughey R (2011) Core-glycosylated mucinlike repeats from MUC1 are an apical targeting signal. J Biol Chem 286:39072-39081

Madsen J, Mollenhauer J, Holmskov U (2010) Gp-340/DMBT1 in mucosal innate immunity. Innate Immunity 16:160-167

Marini and Cabada (2003) One step purification and biochemical characterization of a spermatozoa-binding protein from porcine oviductal epithelial cells. Mol Reprod Dev 66:383-390

Mollenhauer J, Herbertz S, Helmke B, Kollender G, Krebs I, Madsen J, Holmskov U, Sorger K, Schmitt L, Wiemann S, Otto HF, Gröne HJ, Poustka A (2001) Deleted in Malignant Brain Tumors 1 is a versatile mucinlike molecule likely to play a differential role in digestive tract cancer. Cancer Res 61: 8880-8886

Müller H, End C, Weiss C, Renner M, Bhandiwad A, Helmke BM, Gassler N, Hafner M, Poustka A, Mollenhauer J, Poeschl J (2007) Respiratory Deleted in Malignant Brain Tumours 1 (DMBT1) levels increase during lung maturation and infection. Clin Exp Immunol 151:123-129 
Müller H, Hu J, Popp R, Schmidt M, Müller-Decker K, Mollenhauer J, Fisslthaler B, Eble J, Fleming I (2012) Deleted in Malignant Brain Tumors 1 is present in the vascular extracellular matrix and promotes angiogenesis. Arterioscler Thromb Vasc Biol 32:442-448

Pérez FA, Roma S, Cabada M, Marini PE (2006) Sperm binding glycoprotein is differentially present surrounding the lumen of isthmus and ampulla of the pig's oviduct. Anat Embryol 211: 619-624

Renner M, Bergmann G, Krebs I, End C, Lyer S, Hilberg F, Helmke B, Gassler N, Autschbach F, Bikker F, Strobel-Freidekind O, Gronert-Sum S, Benner A, Blaich S, Wittig R, Hudler M, Ligtenberg AJ, Madsen J, Holmskov U, Annese V, Latiano A, Schirmacher P, Amerongen AV, D'Amato M, Kioschis P, Hafner M, Poustka A, Mollenhauer J (2007) DMBT1 confers mucosal protection in vivo and a deletion variant is associated with Crohn's disease. Gastroenterology 133:1499-1509

Rossez Y, Coddeville B, Elass E, Quinchon J, Vidal O, Corfield AP, Gosset P, Lacroix J, Michalski J, Robbe-Masselot C (2011) Interaction between DMBT1 and galectin 3 is modulated by the structure of the oligosaccharides carried by DMBT1. Biochimie 93:593-603

Schwarzer C, Esteves T, Araúzo-Bravo M, Le Gac S, Nordhoff V, Schlatt S, Boiani M (2012) ART culture conditions change the probability of mouse embryo gestation through defined cellular and molecular responses. Hum Reprod 27:2627-2640

Stoddard E, Cannon G, Ni H, Karikó K, Capodici J, Malamud D, Weissman D (2007) gp340 expressed on human genital epithelia binds HIV-1 envelope protein and facilitates viral transmission. J Immunol 179:31263132

Straube T, von Mach T, Hönig E, Greb C, Schneider D, Jacob R (2013) pH-Dependent Recycling of Galectin3 at the Apical Membrane of Epithelial Cells. Traffic doi: 10.1111/tra.12086

Takito J, Yan L, Ma J, Hikita C, Vijayakumar S, Warburton D, Al-Awqati Q (1999) Hensin, the polarity reversal protein, is encoded by DMBT1, a gene frequently deleted in malignant gliomas. Am J Physiol 277:F277-289 
Takito J, Al-Awqati Q (2004) Conversion of ES cells to columnar epithelia by hensin and to squamous epithelia by laminin. J Cell Biol 166:1093-1102

Teijeiro J, Cabada M, Marini PE (2008) Sperm Binding Glycoprotein (SBG) produces calcium and bicarbonate dependent alteration of acrosome morphology and protein tyrosine phosphorylation on boar sperm. J Cell Biochem 103:1413-1423

Teijeiro J, Dapino D, Marini PE (2011) Porcine oviduct sperm binding glycoprotein and its deleterious effect on sperm: a mechanism for negative selection of sperm? Biol Res 44: 329-337

Teijeiro J, Marini PE (2012) The effect of oviductal deleted in malignant brain tumor 1 over porcine sperm is mediated by a signal transduction pathway that involves pro-AKAP4 phosphorylation Reproduction 143:773785

Teijeiro J, Roldán M, Marini PE (2012) Molecular identification of the sperm selection involved porcine sperm binding glycoprotein (SBG) as deleted in malignant brain tumors 1 (DMBT1) Biochimie 94:263-267

Tynan S, Pacia E, Haynes-Johnson D, Lawrence D, D'Andrea MR, Guo JZ, Lundeen S, Allan G (2005) The putative tumor suppressor deleted in malignant brain tumors 1 is an estrogen-regulated gene in rodent and primate endometrial epithelium. Endocrinology 146:1066-1073

Vasta GR, Ahmed H, Nita-Lazar M, Banerjee A, Pasek M, Shridhar S, Guha P, Fernández-Robledo JA (2012) Galectins as self/non-self recognition receptors in innate and adaptive immunity: an unresolved paradox. Front Immunol 3:199 doi:10.3389/fimmu.2012.00199

Vijayakumar S, Erdjument-Bromage H, Tempst P, Al-Awqati Q (2008) Role of Integrins in the Assembly and Function of Hensin in Intercalated Cells. J Am Soc Nephrol 19:1079-1091

Vijayakumar S, Takito J, Hikita C, Al-Awqati Q (1999) Hensin remodels the apical cytoskeleton and induces columnarization of intercalated epithelial cells: processes that resemble terminal differentiation. J Cell Biol 144:1057-1067

von Wolff M, Wang X, Gabius H, Strowitzki T (2005) Galectin fingerprinting in human endometrium and decidua during the menstrual cycle and in early gestation. Mol Hum Reprod 11:189-194 
Yang H, Lei C, Cheng C, Feng Y, Zhang W, Petracco RG, Sak S (2012) The antiapoptotic effect of galectin-3 in human endometrial cells under the regulation of estrogen and progesterone. Biol Reprod 87:39-47

Zumoffen C, Gil R, Caille A, Morente C, Munuce M, Ghersevich SA (2013) A protein isolated from human oviductal tissue in vitro secretion, identified as human lactoferrin, interacts with spermatozoa and oocytes and modulates gamete interaction. Hum Reprod 28:1297-1308

\section{Figure Captions}

Fig. 1 Expression of DMBT1 in the Fallopian tube. (a) Western blot of Fallopian tube extracts, after 8\% SDS-PAGE, with anti-CUB and anti-hDMBT1p84 antibodies. Both polyclonal antibodies recognize DMBT1. Immunohistochemistry of Fallopian tube with (b) anti-CUB or (c) anti-hDMBT1p84 antibodies, and DAB staining. DMBT1 localizes at the surface of the human oviductal epithelial cells (d) Incubation with rabbit pre-immune serum as negative control. Bars indicate $10 \mu \mathrm{m}$.

Fig. 2 Expression of gal-3 in porcine oviducts. (a) Western blot of porcine (PE) and human (HE) oviductal cell extracts, after 12\% SDS-PAGE, with anti-gal-3 antibodies. The $31 \mathrm{kDa}$ gal-3 protein is recognized in both samples. Immunohistochemistry of porcine oviduct with (b) anti-gal-3 antibodies and DAB staining. Gal-3 localizes at the surface of the porcine oviductal epithelial cells and staining of some nuclei is also detected. (c) Negative control. Bars indicate $10 \mu \mathrm{m}$.

Fig. 3 Co-localization of DMBT1 and gal-3 in the human and porcine oviducts. Fallopian tubes: (a) Hoechst 33258 staining (blue, nuclei); (b) DMBT1 fluorescent (cy2, green) detection, intense expression of DMBT1 at the apical surface of the cells and granular diffuse staining distributed over the entire cytoplasm is seen, in the crypts bases as well as in the more luminal cells; (c) gal-3 fluorescent detection (cy3, red), expression of gal-3 is seen predominantly at the apical surface of the cells and at occasional nuclei; (d) merge of images $\mathbf{a}, \mathbf{b}$ and c; (e) square enlargement indicated in d, showing the same location for DMBT1 and galectin-3 (yellow); (f) bright field of micrograph e. Gilts oviducts: (g) Hoechst 33258 staining (blue, nuclei); (h) immunoreactivity of DMBT1 (cy2, green) localized predominately at the apical membrane of the epithelium, and was also present as a diffuse pattern over the cytoplasm, the basal periphery of some cells showed staining; (i) 
immunorectivity for gal-3 (cy3, red) localizes primarily at the apical membrane of the epithelial cells, and some cells also show mild supranuclear and cytoplasmic fluorescence; (j) merge of images $\mathbf{g}, \mathbf{h}$ and $\mathbf{i}$, showing co-localization at the apical surface (yellow); (k) enlargement of the square indicated in $\mathbf{j}$; (l) bright field of micrograph $\mathbf{k}$. Immunofluorescence of human (m) and porcine (n) oviducts with pre-immune serum. For DMBT1 detection anti-CUB antibodies were used. Scale bars: a-d, g-j and $\mathbf{n}: 50 \mu \mathrm{m}$; e-f, k-l and m: 20 $\mu \mathrm{m}$.

Fig. 4 Co-immunoprecipitation assay for DMBT1 and galectin-3. (a) Immunoprecipitation with anti-gal-3 antibodies (IP: a-gal-3) followed by western blot (WB) with anti-CUB (top) and anti-gal-3 antibodies (bottom); DMBT1 co-immunoprecipitates with gal-3. (b) Immunoprecipitation with anti-CUB antibodies (IP: a-CUB) followed by western blot with anti-CUB (top) and anti-gal-3 antibodies (bottom); galectin-3 coimmunoprecipitates with DMBT1. C+: membrane rich fraction from oviductal cells; W1-2-3: washes; IP: immunoprecipitated proteins. 


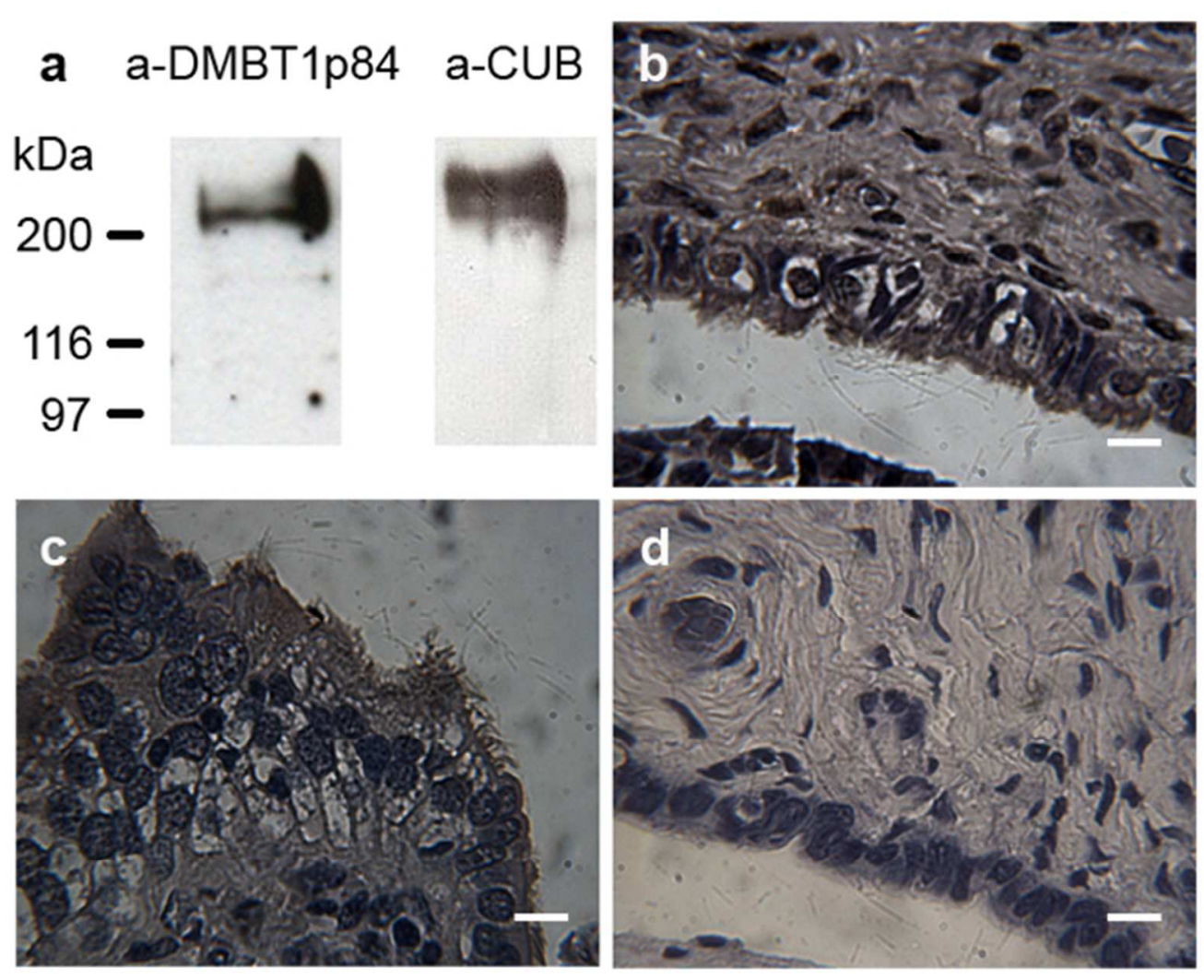

Fig. 1

$83 \times 67 \mathrm{~mm}(200 \times 200$ DPI $)$ 

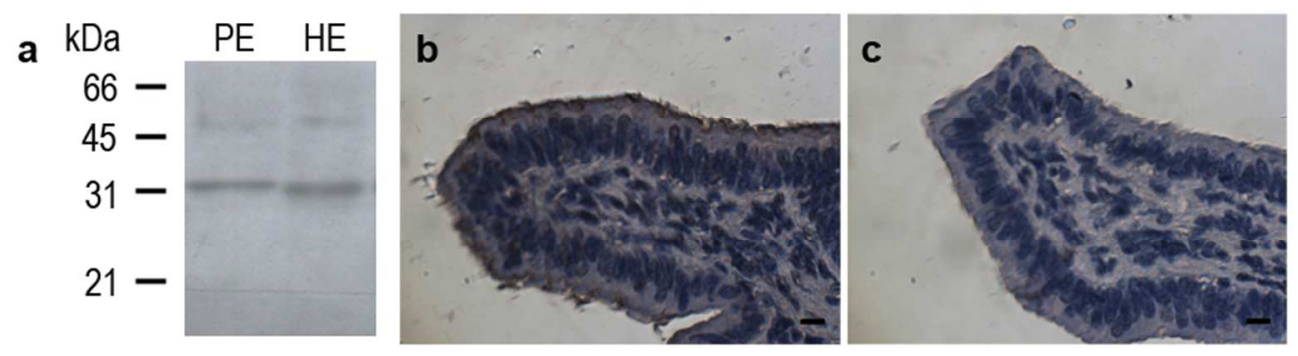

$120 \times 33 \mathrm{~mm}(200 \times 200$ DPI $)$

2

3

5

6

8

9

10

11

13

14
15

16

17

18

19

20

21

22

23

24

25

26

27

28

29

30

31

32

33

34

35

36

37

38

39

40

41

42

43

44

45

46

47

48

49

50

51

52

53

54

55

56

57

58

59

60 


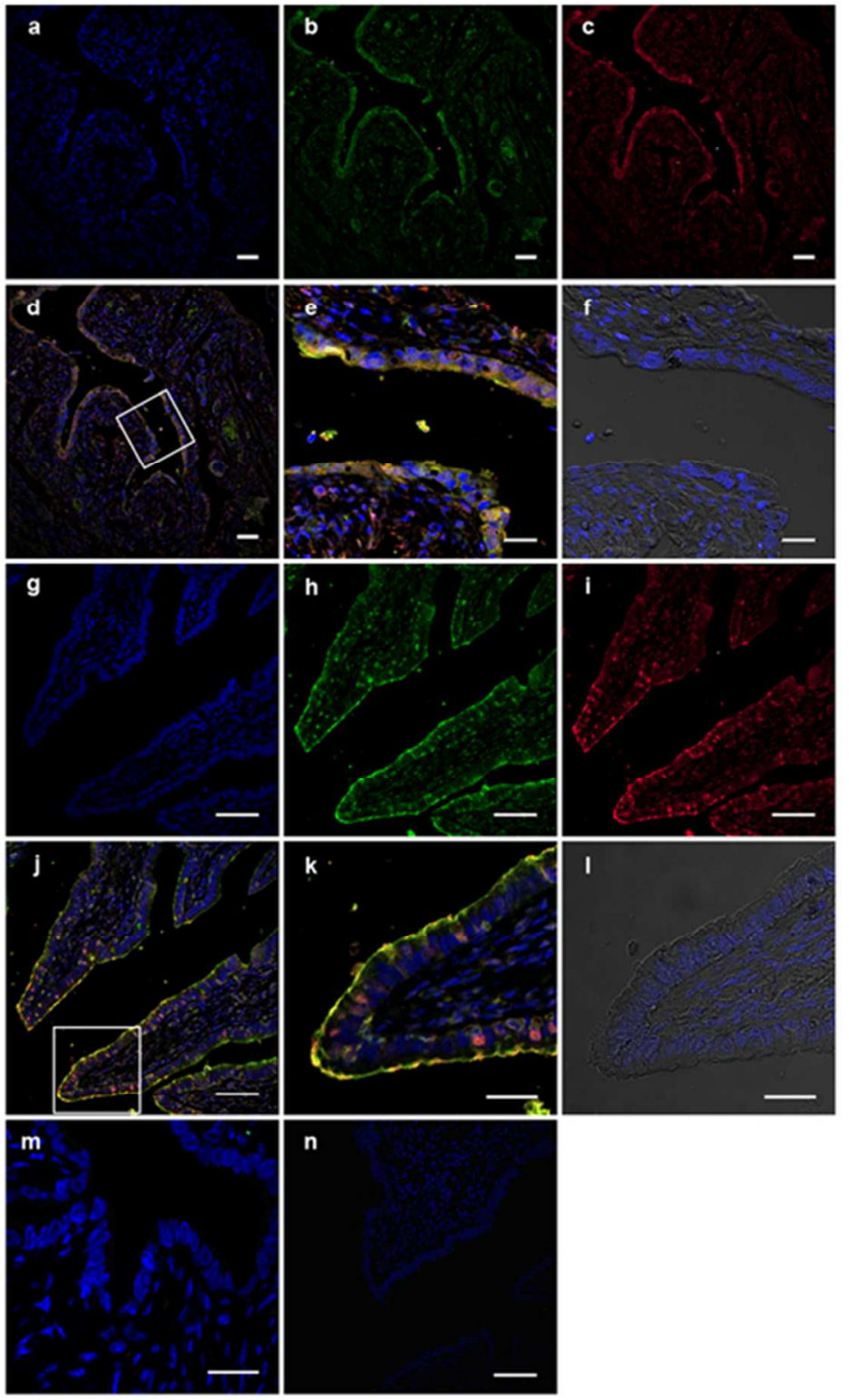

$173 \times 289 \mathrm{~mm}(72 \times 72 \mathrm{DPI})$ 
a

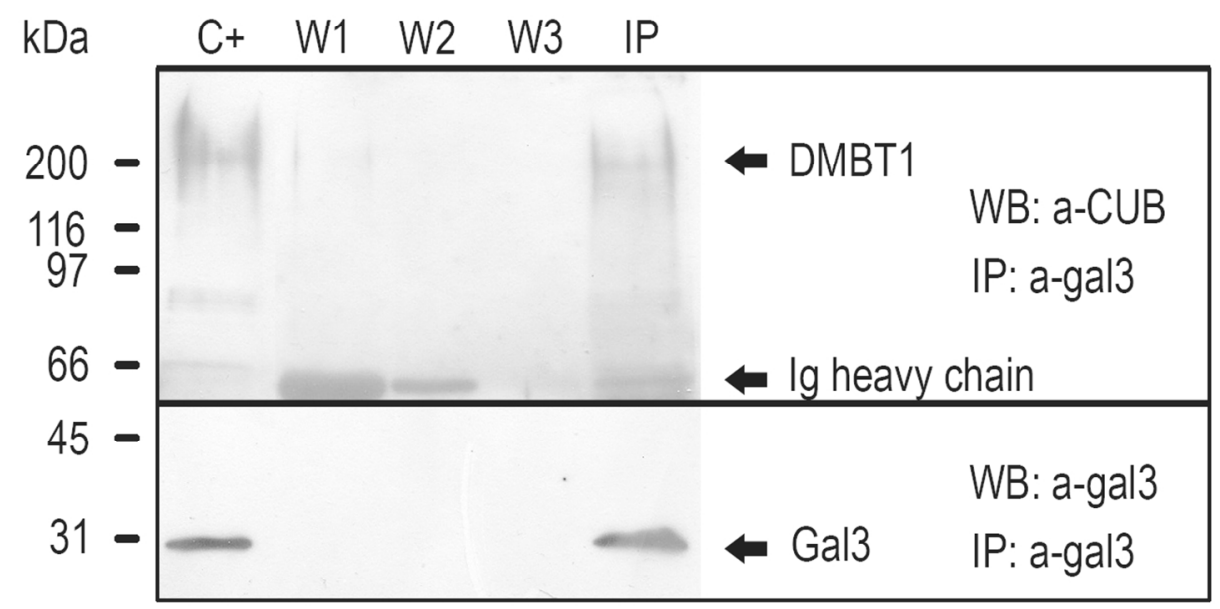

b

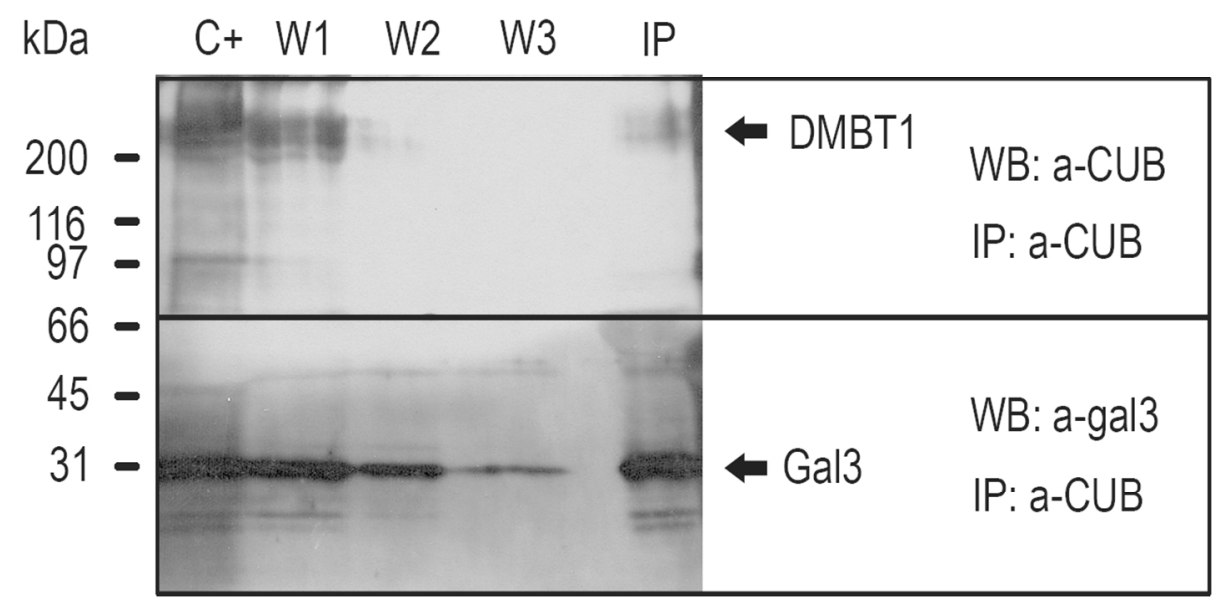

$176 \times 206 \mathrm{~mm}(200 \times 200$ DPI $)$ 